\title{
Processing of the Replicase of Murine Coronavirus: Papain-like Proteinase 2 (PLP2) Acts to Generate p150 and p44
}

\author{
AMORNRAT KANJANAHALUETHAI AND SUSAN C. BAKER \\ Department of Microbiology and Immunology, Loyala University of Chicago, Stritch School \\ of Medicine, Maywood, IL USA
}

\section{INTRODUCTION}

For Nidoviruses, proteolytic processing of a large polyprotein translated from the 5'-end of the genomic RNA is required for the maturation and assembly of the viral replicase complex. The scheme used to process the arterivirus equine arteritis virus (EAV) replicase polyprotein has been experimentally determined (reviewed in Snijder and Meulenberg, 1998). The EAV replicase polyprotein is processed by 3 distinct proteinases to generate 12 mature protein products. Polyprotein intermediates have been identified as well as major and minor processing pathways. However, the role of the intermediates and the alternate pathways remains to be investigated. For the coronavirus mouse hepatitis virus (MHV), several laboratories are investigating the pathways used to process the replicase polyprotein. Two major processing models have been postulated and are shown in Figure 1. Studies from our laboratory showed that p150 is an intermediate to the $3 \mathrm{C}$-like proteinase (3CLpro) product $\mathrm{p} 27$ and that $\mathrm{p} 150$ likely extends to include the putative membrane-spanning protein domain 1, MP1 (Schiller et al 1998). In contrast, other investigators have not detected the p150 precursor and postulate that a $\mathrm{p} 240$ product is adjacent to p27 (Denison et al 1992; 1995 and Lu et al 1998). In this study, we developed a specific antiserum to the MP1 domain (anti-D11) and determined that the 
MP1 domain is indeed part of the p150 intermediate. Furthermore, we show that MHV papain-like proteinase 2 (PLP2) is responsible for cleaving the polyprotein at the putative 150 cleavage site. These results show that PLP2 is an active enzyme.

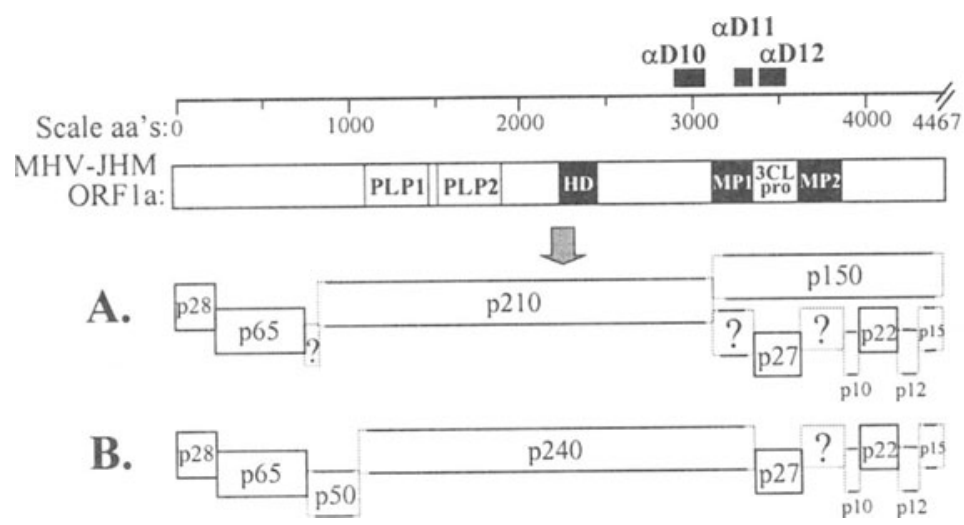

Figure I. Two models for the proteolytic processing of the MHV ORF1 a polyprotein. Model A is modified from Schiller et al (1998) and depicts the p150 precursor to the 3CLpro product. Model B is from the data of Denison et al (1992 and 1995) and Lu et al (1998) and indicates that the MP1 domain is part of the $\mathrm{p} 240$ protein.

\section{MATERIALS AND METHODS}

\subsection{Virus, cell line and immunoprecipitation}

The MHV-JHM-X strain of coronavirus was used to infect HeLa-MHVR cells at moi 1 . Infected cells were radiolabled with $100 \mu \mathrm{Ci} / \mathrm{ml}\left({ }^{35} \mathrm{~S}\right)$ translabel from 5 to $7 \mathrm{~h}$ post-infection (pi). Lysates were prepared and subjected to immunoprecipitation as previous described by our laboratory (Schiller et al 1998). The polyclonal rabbit antisera were generated after injection of GST-MHV fusion proteins and the specificity of the antisera was demonstrated as previously described (anti-D10 and anti-D12 in Schiller et al 1998; anti-D11 in Kanjanahaluethai and Baker, 2000). The anti-V5 
monoclonal antibody (Invitrogen) is directed against an epitope tag built into the constructs.

\subsection{Transfection of plasmid DNA and vTF7.3-mediated expression of MHV replicase products}

Plasmid DNAs encoding the PLP2 domain and putative p150 cleavage site (substrate) under control of the $\mathrm{T} 7$ promoter were generated and expressed via the vaccinia-T7 system (Fuerst et al 1978) as described in detail by Kanajanahaluethai and Baker (2000). Newly synthesized proteins were radiolabeled with $50 \mu \mathrm{Ci} / \mathrm{ml}\left({ }^{35} \mathrm{~S}\right)$-translabel from 5.5 to $10.5 \mathrm{~h}$ pi and lysates were prepared and subjected to immuno-precipitation with the designated antisera. Products were analyzed by $5-10 \%$ SDS-PAGE.

\section{RESULTS AND DISCUSSION}

Previously, we hypothesized that the MP1 domain was part of the p150 intermediate generated during the processing of the MHV replicase polyprotein (Schiller et al 1998). To test this hypothesis, we generated a specific antiserum, anti-D11, and used it to immunoprecipitate replicase products from MHV-infected cells. We compared the products immunoprecipitated with anti-D11 to products detected by antisera directed against a region upstream of MP1 (D10 region) and the region downstream of MP1 (the 3CLpro region precipitated by anti-D12). As shown in Figure 2, antiD11 sera precipitates MHV specific products of $>300$ kilodaltons $(\mathrm{kDa}), 150$ $\mathrm{kDa}$ and $44 \mathrm{kDa}$ (lane 4). The 150 product migrates slightly faster than a product detected with all preimmune and immune sera tested (indicated by the open arrow), and co-migrates with the 150 product identified by antiD12 (Schiller et al 1998). The p150 product is clearly distinct from the p210 and p290 proteins detected with anti-D10 (compare lane 2 with lanes 4 and 6). These results indicate that the p150 intermediate does indeed encompass the MP1 domain. Furthermore, pulse-chase analysis and proteinase inhibition studies indicate that $\mathrm{p} 150$ is a precursor to $\mathrm{p} 44$ and $\mathrm{p} 27$, and that the proteinase responsible for generating p150 is not sensitive to proteinase inhibitor E64d (Kanjanahaluethai and Baker, 2000). 


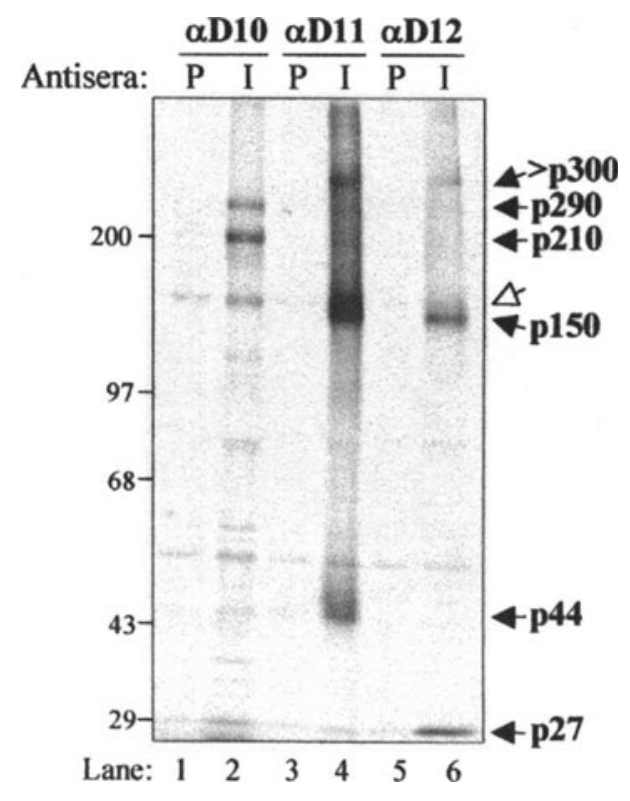

Figure 2. Detection of replicase gene products from MHV-infected HeLa-MHVR cells. Viral products detected by immunoprecipitation using preimmune (P) and immune (I) sera were separated by $5-10 \%$ SDS-PAGE. MHV replicase gene products $>$ p300, p290, p210, $\mathrm{p} 150, \mathrm{p} 44$ and $\mathrm{p} 27$ are indicated by filled arrows. A non-specific product co-precipitated at low level by both preimmune and immune sera is indicated by the open arrow.

To identify the proteinase domain responsible for cleaving p150, we generated constructs encoding a single proteinase domain (PLP1, PLP2 or 3CLpro) and a construct encoding the putative p150 cleavage site (the substrate), and tested for the ability of the proteinase domain to cleave the substrate in trans. We found that the protein encoded by the PLP2 domain was indeed an active proteinase and rapidly cleaved the substrate to generate p44 (Figure 3). Neither the PLP1 nor the 3CLpro domains acted in trans to efficiently process the substrate (data not shown). These results show that the previously predicted PLP2 domain is an active proteinase and acts to process the precursor polyprotein to generate the p150 intermediate. P150 is likely then processed by the encoded 3CLpro domain to generate $\mathrm{p} 44, \mathrm{p} 27$, p10, p22, p12, p15 and the MP2 product (Figure 4A).

The p44 product identified in this study is analogus to the nsp3 replicase product of EAV (van der Meer et al 1998) and the $41 \mathrm{kDa}$ product recently identified for avian infectious bronchitis virus (IBV) (Lim et al 2000). The $41 \mathrm{kDa}$ IBV replicase product was shown to be modified by $\mathrm{N}$ linked glycosylation. It will be interesting to determine if the MHV p44 replicase product is also glycosylated. 


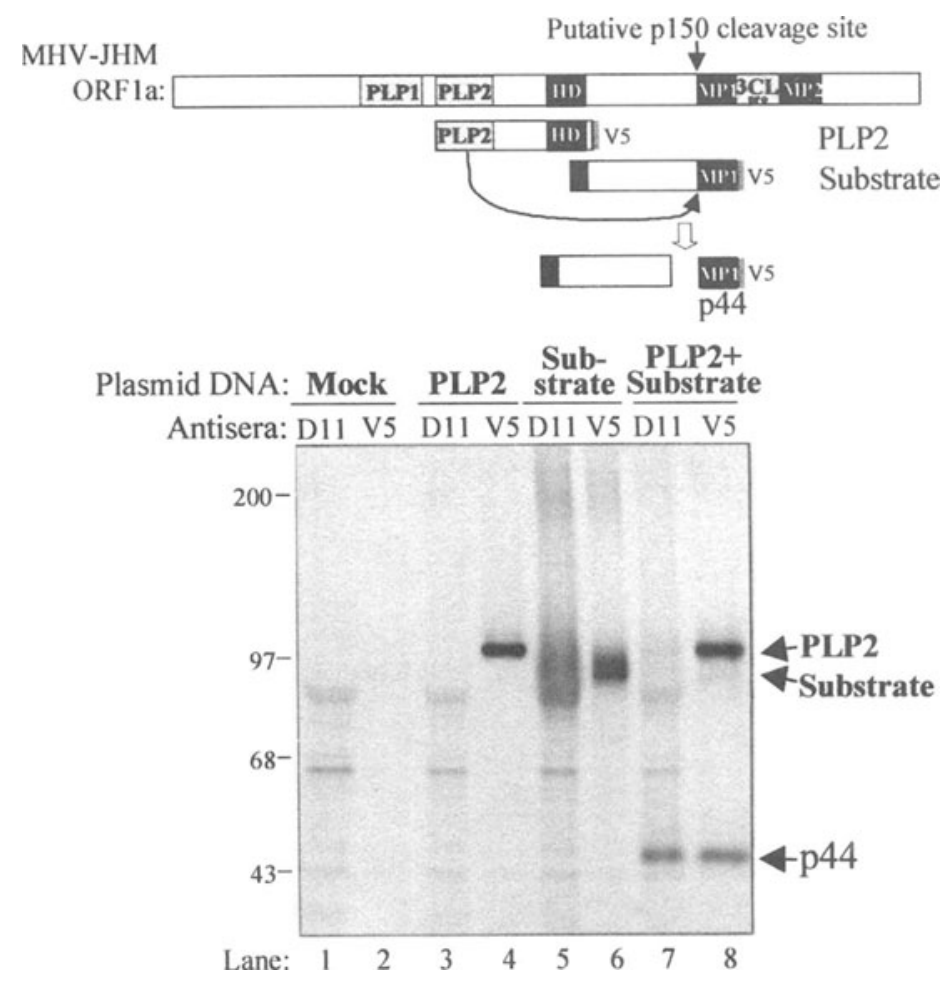

Figure 3. Detection of trans-cleavage activity by MHV PLP2. Plasmids encoding the PLP2 domain and the putative p150 cleavage site were transfected (either independently or together) into vTF7.3-infected HeLa-MHVR cells, newly synthesized proteins were radiolabeled and subjected to immunoprecipitation with the indicated antisera, and analyzed by $5-10 \%$ SDS-PAGE. The PLP2, full length substrate and p44 cleavage products are indicated by arrows.

The processing of p150 may be critical for releasing a hydrophobic sequence (MP1) that acts as an important membrane anchor for the MHV replicase complex (Figure 4B). We speculate that early processing events such as the cleavage of p 28 by PLP1 and cleavage of p 150 by PLP 2 occur co-translationally in cis and are required to direct the intermediates encoding the hydrophobic domains to become membrane associated. This membrane association may drive the formation of the MHV double membrane vesicles (DMVs) (van der Meer et al 1998; Gosert et al 2000). The DMVs are likely the sites where MHV RNA synthesis occurs. The processing intermediates may serve as the replicase complex that mediates negative strand RNA synthesis, whereas further processing may be required to allow the complex to function in positive strand RNA synthesis. This scenario is based in part on our knowledge of the role of proteolytic processing in the switch from 
negative strand RNA synthesis to positive strand RNA synthesis in alphaviruses such as Sindbis virus (Lemm et al 1994; Shirako and Strauss, 1994). In that system, the nsP2 proteinase acts initially in cis and then in trans to process the replicase precursor from the P123-nsP4 complex responsible for negative strand RNA synthesis to the nsP1, nsP2, nsP3, nsP4 complex that mediates positive strand RNA synthesis. It will be interesting to determine if proteolytic processing of the MHV replicase complex is required for a similar switch from negative to positive strand RNA synthesis.

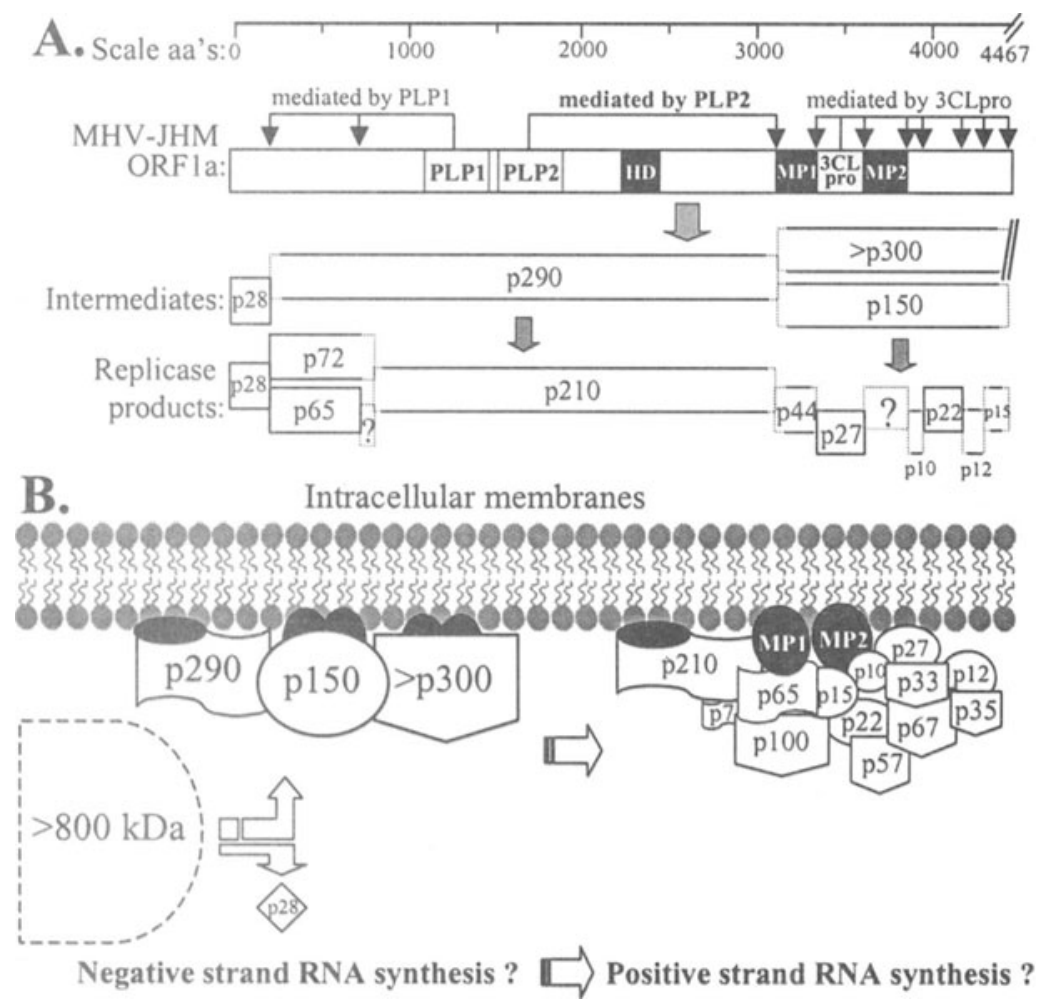

Figure 4. A) Schematic diagram of MHV ORFla replicase intermediates and products. The proteinase domain that mediates each cleavage event is indicated. B) Working model for MHV replicase membrane association, assembly and function. All putative ORFla and ORF $1 \mathrm{~b}$ products are indicated.

\section{ACKNOWLEDGEMENTS}

We thank David Axtell for his assistance in the production of GST-fusion protein and John Zaryczny for his assistance with rabbit injection and sera collection. This work was supported by Public Health Service Research Grant AI 32065. 


\section{REFERENCES}

Denison, M.R., Hughes, S.A., and Weiss, S.R. 1995. Identification and characterization of a $65-\mathrm{kDa}$ protein processed from the gene 1 polyprotein of the murine coronavirus MHVA59. Virology 207:316-320.

Denison, M.R., Zoltick, P.W., Hughes, S.A., Giangreco, B., Olson, A.L., Perlman, S., Leibowitz, L.L., and Weiss, S.R. 1992. Intracellular processing of the N-terminal ORFla proteins of the coronavirus MHV-A59 requires multiple proteolytic events. Virology 189:274-284.

Fuerst, T.R., Niles, E.G., Studier, F.W., and Moss, B. 1986. Eukaryotic transient-expression system based on recombinant vaccinia virus that synthesizes bacteriophage T7 RNA polymerase. Proc. Natl. Acad. Sci. USA. 83:8122-8126.

Gosert, R., Kanjanahaluethai, A., Egger, D., Bienz, K., and Baker, S.C. 2000. Comparison of replicase localization in different types of mouse hepatitis virus (MHV)-infected cells. In The Nidoviruses. (E. Lavi, ed.), Plenum Press, New York (in press).

Kanjanahaluethai, A., and Baker, S.C. 2000. Identification of mouse hepatitis virus papainlike proteinase 2 activity. J. Virol. (in press)

Lemm, J.A., Rumenapf, T., Strauss, E.G., Strauss, J.H., and Rice, C.M. 1994. Polypeptide requirements for assembly of functional Sindbis virus replication complexes: A model for the temporal regulation of minus- and plus-strand RNA synthesis. EMBO J. 13: 2925-2934.

Lim, K.P., Ng, L.F.P., and Liu, D.X. 2000. Identification of a novel cleavage activity of the first papain-like proteinase domain encoded by open reading frame la of the coronavirus avian infectious bronchitis virus and characterization of the cleavage products. J. Virol. 74:1674-1685.

Lu, Y., Sims, A.C., and Denison, M.R. 1998. Mouse hepatitis virus 3C-like protease cleaves a 22-kilodalton protein from the open reading frame la polyprotein in virus-infected cells and in vitro. J. Virol. 72:2265-2271.

Schiller, J.J., Kanjanahaluethai, A., and Baker, S.C. 1998. Processing of the coronavirus MHV-JHM polymerase polyprotein: Identification of precursors and proteolytic products spanning 400 kilodaltons of ORF1a. Virology 242:288-302.

Skirako, Y., and Strauss, J.H. 1994. Regulation of Sindbis virus RNA replication: Uncleaved P123 and nsP4 function in minus-strand RNA synthesis, whereas cleaved products from P123 are required for efficient plus-strand RNA synthesis. J.Virol. 68:1874-1885.

Snijder, E.J., and Meulenberg, J.J.M. 1998. The molecular biology of arteriviruses. J. Gen. Virol. 79:961-979.

van der Meer, Y., van Tol, H., Locker, J.K., and Snijder, E.J. 1998. ORF1 a-encoded replicase subunits are involved in the membrane association of the arterivirus replication complex. J. Virol. 72:6689-6698. 